\title{
LAS AUDIENCIAS VIRTUALES \\ ANÁLISIS DE SUS VENTAJAS Y LIMITACIONES \\ EN LA PRÁCTICA DE PRUEBAS TESTIFICAL, \\ PERICIAL Y LA DECLARACIÓN DEL IMPUTADO*
}

\author{
Reyler Rodríguez Chávez ${ }^{* *}$ \\ Universidad Católica Sedes Sapientiae \\ reyler6@gmail.com
}

Resumen: Las audiencias virtuales constituyen hoy en día una muestra de la real expresión que viene ejerciendo la tecnología en el campo del proceso, respondiendo no solo a una exigencia coyuntural, sino también, a la necesidad concreta de resolver distintas situaciones como la inasistencia de las partes, las distancias, impedimentos físicos, entre otros, que tienen como común denominador, la frustración de determinadas pruebas como son la declaración testimonial, pericial o la declaración del imputado. Frente a estas circunstancias adversas para el desarrollo de la actividad probatoria, el empleo de instrumentos como la videoconferencia parece ofrecer una alternativa útil que hace viable la interconexión de las partes, los abogados, el fiscal, los peritos, el juez, y de todos aquellos cuya participación en el proceso resulte relevante. Sin embargo, se presentan ciertas cuestiones que podrían resultar limitativas como el derecho de defensa, la inmediación, la privacidad de los datos e información reservada, las garantías de veracidad y legitimidad, entre otros, cuya tutela debe garantizarse en todo proceso que incorpore este tipo de espacios virtuales.

Palabras clave: Las TIC, audiencia virtual, videoconferencia, prueba testimonial, prueba pericial, declaración del imputado, imparcialidad, inmediación, derecho de defensa,

\footnotetext{
* Ensayo presentado en el Máster en Derecho, Empresa y Justicia de la Universidad de Valencia, España, a la cátedra denominada Líneas Generales para una Modernización de la Justicia en un Mundo Globalizado: Derecho, Proceso y Seguridad Jurídica, dirigido por la profesora Ana Isabel Blanco García.

** Profesor contratado de la Facultad de Derecho de la Universidad Católica Sedes Sapientiae (UCSS). Presidente del Consejo Consultivo Internacional de IusTech. Miembro honorario de E-Justicia Latinoamericana. Máster en Argumentación Jurídica por la Universidad de Alicante (España) y por la Universidad de Palermo (Italia). Magíster en Derecho de la Empresa por la Pontificia Universidad Católica del Perú. Estudios del Máster en Derecho, Empresa y Justicia por la Universidad de Valencia (España). Abogado por la Universidad Nacional de Cajamarca.
} 
privacidad de datos, seguridad digital.

\title{
THE VIRTUAL HEARINGS. ANALYSIS OF ITS ADVANTAGES AND LIMITA- TIONS IN THE PRACTICE OF TESTIFICAL, EXPERT EVIDENCE AND THE DECLARATION OF THE IMPUTE
}

\begin{abstract}
Virtual audiences are today a sample of the real expression that technology has been exercising in the process field, responding not only to a conjuncture demand, but also to the specific need to resolve different situations such as the absence of the parties, distances, physical impediments, among others, which have as a common denominator, the frustration of certain tests such as the testimonial, expert or the statement of the accused. Faced with these adverse circumstances for the development of the evidentiary activity, the use of instruments such as videoconferencing seems to offer a useful alternative, which makes viable the interconnection of the parties, the lawyers, the prosecutor, the experts, the judge, and all those whose participation in the process is relevant. However, certain issues are presented that could be limiting such as the right of defense, immediacy, privacy of data and reserved information, guarantees of veracity and legitimacy, among others, whose protection must be guaranteed in any process that incorporates this type of virtual spaces.
\end{abstract}

Keywords: ICT, Virtual Hearing, Videoconference, Testimonial Evidence, Expert Evidence, Statement of the Accused, Impartiality, Immediacy, Right of Defense, Data Privacy, Digital Security.

\section{Introducción}

Al día de hoy, asistimos a un nuevo escenario donde cualquier forma de comunicación es posible gracias al empleo de las Tecnologías de la Información y la Comunicación (en adelante, las TIC) en el ámbito jurídico. La sociedad contemporánea ha recepcionado estos nuevos avances sea por necesidad, por innovación o por la simple invasión de las tecnologías en los diversos espacios. Sin mayores reparos, lo cierto es que vivimos bajo un contexto donde prima el uso de los medios tecnológicos y cuya presencia se ha extendido a todo el sistema de justicia, y en particular, al ámbito del proceso judicial, especialmente en el campo de la actuación de las pruebas testimonial, pericial y la declaración de imputado. 
Una de las virtudes de la llamada era de las tecnologías, es la de facilitar la comunicación entre los seres humanos. Aquello supone eliminar las barreras que impedían esta finalidad como las distancias, el tiempo, la velocidad, la fluidez y claridad de la comunicación, entre otros factores. La expansión del mundo globalizado es el evidente resultado de la influencia de las TIC y del despliegue de todo su poder para lograr la interconexión mundial. Frente a este escenario, señala Blázquez (2001, pp. 18, 20), que estaríamos ahora en la cuarta era o revolución cuyos ejes están constituidos por la información y la comunicación y cuyo soporte son elementos electrónicos, agrega que, la "interactividad" es una de las características más potente de las nuevas tecnologías que permite emitir y recibir información, así como dialogar, conversar y transmitir información y conocimientos sin límite de distancia ni de tiempo.

Esta profunda trasformación de la sociedad en general supone la hegemonía del hombre digital que se mueve y se adecua al rito que impone la tecnología. De manera que la comunicación por medio de los recursos tecnológicos es una exigencia producto de un modo de vida impuesto por el trabajo, las relaciones personales, familiares, o simplemente por la tendencia social. Ortega y Gasset (1929) en su magnífica obra La rebelión de las masas, advertía la presencia del hombre masa como protagonista de las relaciones masivas y de la sociedad de masas que resultó a principios del siglo XX con el advenimiento de las máquinas. Acontece ahora la era de las TIC, y con ello, el surgimiento de una sociedad en red, digital, informática, donde el hombre virtual emerge como actor principal en todo tipo de relaciones acaecidas en este contexto. Sotomarino (2010, p. 9) señala que el manejo intensivo de las generaciones que han nacido o se han desarrollado gozando las ventajas de la comunicación a través de correos electrónicos y páginas web, tienen las características de nativos digitales al nacer y desarrollarse en dicho medio

Lo que importa hoy en día es estar interconectado, formar redes, y sentir que se tiene cierto poder para acceder a la información y comunicación de manera rápida, sencilla y sin costos. El hombre digital es un ser omnipresente.

Sin embargo, toda evolución trae consigo nuevas preocupaciones y responsabilidades, tanto en el ámbito personal como social, así como la aparición de nuevas formas o modalidades de afectación de los bienes y derechos que son objeto de titularidad en el contexto de la sociedad tecnológica. Al respecto, Pérez Luño resalta que el impacto de las NT (Nuevas Tecnologías) en el ámbito de los derechos humanos representa un fenómeno ambivalente:

...de una parte, las NT han determinado la aparición de nuevos derechos que suponen la ampliación o profundización de determinados espacios de libertad; de 
otra, han contribuido a la emergencia de derechos cuya finalidad es la garantía de los ciudadanos frente a un desarrollo tecnológico liberticida. (2010, p. 35)

Asistimos a un nuevo escenario virtual, un espacio caracterizado por la relatividad del tiempo y del espacio, aunado al control de la velocidad en el intercambio y cantidad de información. Es un nuevo espacio donde las relaciones interpersonales van quedando de lado para dar paso a las relaciones virtuales, a larga distancia o telemáticas. Ello es posible gracias a las TIC, tecnologías que permiten esta importante interacción y se centran especialmente en el tratamiento de la información por medios digitales.

Al respecto, Díaz (2010) señala lo siguiente:

Consideramos como «tecnologías de la información y de la comunicación» o «nuevas tecnologías» (que en este contexto usaremos como sinónimos), $\mathrm{y}$ entre otros instrumentos de transmisión y recepción de comunicaciones e informaciones, la telefonía fija, el móvil, la radio y la televisión, el GPS (Short Message Service) y otros servicios de mensajería, el correo electrónico, el chat o mensajería instantánea, y ocupando un indiscutible protagonismo, Internet, que incluye hoy buena parte de las anteriores prestaciones. (pp. 35-36)

Como podemos apreciar, las TIC comprenden una seria de mecanismos y medios cuya naturaleza común es el empleo de las tecnologías como vehículo para el transporte e intercambio de la información de una manera rápida, en tiempo real, y sin necesidad de hacerlo en forma presencial. Pues basta la existencia de canales tecnológicos que permiten el envío y recepción de información en tiempo real, incluso estando a extremos del planeta.

Las TIC hoy en día constituyen una invención sin la cual el mundo de la comunicación y todo lugar donde sea necesario intercambiar y procesar información de distinta índole, no puede marchar con normalidad. Las TIC son la herramienta que permite dinamizar el mundo de la información, con lo cual da movimiento a los distintos sistemas públicos y privados de información.

De allí que la Comisión Económica para América Latina y el Caribe ha señalado como características de las TIC (2009):

En primer lugar,...la tecnología...consiste en actividades orientadas a la solución de problemas, que incorporan formas tácitas de conocimiento mediante procesos individuales o institucionales. 
En segundo lugar, los paradigmas se definen sobre la base de un "artefacto" o "dispositivo" que mejora con el transcurso del tiempo y cuya descripción se incluyen sus características tecnológicas y económicas fundamentales.

En tercer lugar,... el carácter local y acumulativo del aprendizaje; por local se entiende la exploración y el desarrollo de nuevas técnicas probablemente se producirán en la proximidad de las técnicas ya utilizadas, en tanto que el término "acumulativo" denota que un nivel de desarrollo tecnológico es producto de experiencias pasadas de producción e innovación y, por ende, el resultado de una secuencia de soluciones a problemas específicos. (p. 28)

Las TIC sin duda implican una nueva era, la era de las tecnologías, la denominada "Era digital" o "e-learning", donde se constituyen en el vehículo de intercambio de información por excelencia. Su influencia es de tal magnitud en la alteración de las relaciones sociales, que ahora las relaciones interpersonales se han convertido también en espacios o foros virtuales. Las redes sociales han reemplazado a las relaciones sociales tradicionales. Y el intercambio de la información se ha virtualizado y digitalizado.

\section{Las TIC y su aplicación al sistema de justicia}

El ámbito jurídico no está exento a este mundo digital o cibernético impuesto por las TIC. De manera que los sistemas y procedimientos en este campo también han tenido que adecuarse a las exigencias de rapidez y eficiencia en el intercambio de la información. Se ha aprovechado el uso de diversos mecanismos tecnológicos que facilitan la virtualidad de la comunicación, haciéndola más accesible para los usuarios, o al menos brindando mayores espacios para el intercambio de la información en diversos ámbitos de la administración pública.

En el sistema español, Mozo y Nogueras (2014), hacen referencia a esta tendencia digital, donde se produce por ejemplo la migración de los documentos de soporte de papel a sus equivalentes en formato electrónico. Una de esas manifestaciones ha sido la regulación introducida por la Ley 18/2011, que regula el uso de las tecnologías de la información y comunicación en la Administración de Justicia (LUTICAJ), así como la incorporación de documentos electrónicos regulada por la Ley 1/2000 y el sistema LEXNET.

Otro de los avances en este espacio es la implementación del sistema Lente. Rivielo (2006) señala que el sistema telemático Lente es una plataforma para el intercambio 
seguro de información de gran diversidad de agentes y órganos judiciales, permitiendo la presentación de escritos y documentos, el traslado de copias y la realización de actos de comunicación por vía telemática, garantizando los requisitos exigidos en las leyes procesales.

En otro ámbito, Juan (2015) comentando las modificaciones a la Ley de Enjuiciamiento Civil Española, señala que los medios tecnológicos permiten una serie de ventajas como presentar telemáticamente escritos en las instancias judiciales a cualquier hora del día. Es decir, lo que se conoce como expediente digital, o digitalizado, donde la idea central es la de prescindir del uso del papel físico, y digitalizar todas las actuaciones.

Como podemos apreciar, el uso de las TIC ha sido paulatino, habiendo sido incorporadas para el procesamiento de la información de manera digital. De este modo, el intercambio se hace en forma digital, sin necesidad del empleo de medios físicos como el papel y, por otro lado, el intercambio de la información se realiza de manera interconectada mediante el uso de sistemas o plataformas virtuales como es el caso de Lente.

En países como el Perú, esta vanguardia en el uso de las nuevas tecnologías de la información y comunicación también ha generado importantes modificatorias como es el artículo 234 del Código Civil donde se establece que

...son documentos, los escritos públicos o privados, los impresos, fotocopias, facsímil o fax, planos, cuadros, dibujos, topografías, radiografías, cintas cinematográficas, microformas tanto en la modalidad de microfilm como en la modalidad de soportes informáticos, y otras reproducciones de audio o video, la telemática en general y demás objetos que recojan, contengan o representen algún hecho, o una actividad humana de resultado.

También en el sistema de justicia peruano se ha incorporado el sistema digitalizado a través del expediente electrónico y la notificación electrónica, así como la posibilidad del uso de los medios tecnológicos como las audiencias virtuales.

Como podemos apreciar, las TIC han ganado espacios en todos los sistemas de justicia, facilitando el intercambio de información entre las distintas instancias administrativas $\mathrm{y}$, sobre todo, permitiendo el acceso rápido a la misma por parte de los usuarios de distintos servicios, entre ellos, los del sistema de justicia. 


\section{Ventajas del uso de la videoconferencia en la práctica de pruebas}

El empleo de las TIC en los procesos judiciales, como hemos señalado, se ha dado en distintas modalidades. Estas van desde el empleo de documentos digitalizados a través del expediente digital, el empleo de la notificación electrónica, hasta las agendas virtuales y despachos digitales, entre otros campos.

Montesinos (2009) señala como uno de los empleos de las TIC en el proceso penal, el empleo de las videoconferencias u otros sistemas similares que permitan la comunicación bidireccional y simultánea de la imagen y el sonido para la práctica de determinadas pruebas, así como de otras diligencias. En el sistema español, la Ley de Enjuiciamiento Civil permite en el artículo 299, presentar palabras, datos, cifras y cualquier operación por medio de instrumentos como la filmación, grabación y semejantes.

Uno de los espacios donde las TIC se vienen implementando es el de la videoconferencia en los distintos tipos de diligencias y procesos. La videoconferencia permite la interconexión de personas que se hallan en lugares distintos a través de medios tecnológicos que permiten la interacción de imagen y voz, permitiendo, a su vez, el intercambio de estos elementos. Igualmente, señala que puede haber: a) videoconferencia punto a punto y, b) videoconferencia multipunto, cuando se realiza al emplear dos o más terminales.

Sin duda alguna, el uso de la videoconferencia permite facilitar la actuación de determinadas pruebas como la testimonial, pericial y declaración del imputado. En ese sentido presenta las siguientes ventajas:

a. Facilita la participación de personas que no necesariamente se hallen dentro de la jurisdicción e incluso fuera del país.

b. Genera celeridad procesal, pues no es necesario el desplazamiento de los involucrados, siendo un elemento importante cuando se trata de imputados que se hallan recluidos en un establecimiento penitenciario.

c. Cuando va acompañada de los medios técnicos y personal especializado, otorga inmediación a las actuaciones que se practiquen.

d. El uso de la videoconferencia permite la continuidad de los procesos, evitando que se pueda frustrar o postergar alguna actuación testimonial pericial o declaración.

e. La videoconferencia puede resultar eficiente como mecanismo cuando es necesario la participación simultánea de distintas partes. 
f. También es un medio que en casos complejos y de gravedad, puede permitir proteger la identidad de los testigos.

g. En el caso de las víctimas de delitos especiales como, por ejemplo, los sexuales o donde se afecte la intimidad personal o familiar, la videoconferencia ayuda a proteger la situación de las víctimas al evitar la exposición pública y la victimización secundaria.

De esta manera, la videoconferencia se caracteriza como un instrumento al servicio del proceso cuyas finalidades legales son la protección y agilización de los trámites (Baquero, 2017).

En el caso de la videoconferencia en caso de pruebas testificales, se ha citado la STTS del 17-Marzo-2015 donde se señala que: el recurso a la videoconferencia se encuentra subordinado a la concurrencia de razones de "utilidad" o a la finalidad de evitar que la comparecencia en la sede del órgano ante el que se desarrolle el plenario "resulte gravosa o perjudicial”. (Baquero, 2017).

En este sentido, la videoconferencia, sin duda es un mecanismo que permite celeridad, garantizando la realización de actuaciones que podrían presentar dificultades en cuestiones de presencia física, seguridad y protección de declarantes. De esta forma, la videoconferencia es un medio útil y garante de las actuaciones procesales.

\section{Limitaciones y desventajas del uso de la videoconferencia en la práctica de pruebas}

Sin duda uno de los grandes retos dentro del proceso judicial de la videoconferencia es la garantía del debido proceso, en especial el derecho de defensa de las partes. González (2015) señala que es urgente adaptar la Alecrín a la utilización y exigencias de las nuevas tecnologías, pero sin que su aplicación nos lleve finalmente a infringir evidentes derechos fundamentales. En el mismo sentido, Pérez (2010), señala que en una sociedad como la que nos toca vivir en, la reglamentación jurídica de las NT reviste un interés prioritario, de modo especial, en lo que afecta a la garantía de los derechos humanos.

Baquero (2017) señala que hay limitaciones en la apreciación de la videoconferencia en segunda instancia, lo que podría ocasionar una valoración distinta a la realizada por el juez de primera instancia, afectando así la valoración probatoria. 
Sobre las cuestiones técnicas, Montesinos (2009), en relación a la imagen, señala que se necesitan condiciones mínimas de luminosidad para su correcto funcionamiento y de una correcta disposición física que permita la captación del plano del orador/oyente. En relación al audio, señala que se deberá respetar en todo momento cuidadosamente el tipo de micrófono utilizado, la posición del micrófono frente al orador y, finalmente, la posición del micrófono respecto de lo puntos de sonorización.

Pérez Luño (2010) ha señalado:

Nuestra vida individual y social corren, por tanto, el riesgo de hallarse sometidas a lo que se ha calificado, con razón, de "juicio universal permanente". Ya que, en efecto, cada ciudadano fichado en un banco de datos se halla expuesto a una vigilancia continua e inadvertida, que afecta potencialmente incluso a los aspectos más sensibles de su vida privada; aquellos que en épocas anteriores quedaban fuera de todo control por su variedad y multiplicidad. (p. 21)

Así entonces, el empleo de la videoconferencia deberá cuidar que no se vulneren derechos fundamentales o garantías del debido proceso como el derecho de defensa, la congruencia en la valoración de pruebas en primera y segunda instancia, las cuales permitan el contradictorio, la publicidad y que faciliten la inmediación y apreciación por parte del órgano judicial.

\section{Conclusiones}

a. Las TIC han ganado espacios en todos los sistemas de justicia, facilitando el intercambio de información entre las distintas instancias administrativas y sobre todo permitiendo el acceso rápido a la misma por parte de los usuarios de distintos servicios, entre ellos, los del sistema de justicia.

b. Las audiencias virtuales constituyen hoy en día una muestra de la real expresión que viene ejerciendo la tecnología en el campo del proceso, respondiendo no solo a una exigencia coyuntural, sino también, a la necesidad concreta de resolver distintas situaciones como la inasistencia de las partes, las distancias, impedimentos físicos, entre otros, que tienen como común denominador la frustración de determinadas pruebas, así como la declaración testimonial, pericial o la declaración del imputado. 
c. Las audiencias virtuales ofrecen una alternativa útil, volviendo viable la interconexión de las partes, los abogados, el fiscal, los peritos, el juez, y de todos aquellos cuya participación en el proceso resulte relevante.

d. La videoconferencia, sin duda, es un mecanismo que permite celeridad, garantizando la realización de actuaciones que podrían presentar dificultades en cuestiones de presencia física, seguridad y protección de declarantes. De esta forma, la videoconferencia es un medio útil y garante de las actuaciones procesales.

e. Entre algunas de las deficiencias que se presentan en las videoconferencias se puede señalar las limitaciones al derecho de defensa, la inmediación, la privacidad de los datos e información reservada, las garantías de veracidad y legitimidad, vulneración al derecho de imparcialidad y espontaneidad en las declaraciones, la afectación de la veracidad, entre otros, cuya tutela debe garantizarse en todo proceso que incorpore este tipo de espacios virtuales.

\section{Recomendaciones}

a. El empleo de la videoconferencia deberá cuidar que no se vulnere derechos fundamentales o garantías del debido proceso, tales como el derecho de defensa, la congruencia en la valoración de pruebas en primera y segunda instancia, que permitan el contradictorio, la publicidad y que faciliten la inmediación y apreciación por parte del órgano judicial.

b. Ciertamente, uno de los grandes retos dentro del proceso judicial de la videoconferencia es la garantía del debido proceso, en especial el derecho de defensa de las partes.

c. Deben implementarse módulos de atención para los usuario, los cuales permitan asistirlos y capacitarlos en el acceso a las videoconferencias, reduciendo, además, la brecha digital existente en el desconocimiento y falta de acceso a los medios tecnológicos para acceder a las audiencias por medio de este tipo de mecanismos virtuales. 


\section{Referencias}

Baquero,A. (2017). La videoconferencia en las garantías del proceso penal [Tesis Doctoral, Universidad de Sevilla]. https://idus.us.es/bitstream/handle/11441/67590/ La\%20videoconferencia\%20en\%20las\%20garant\%C3\%ADas\%20del\%20 proceso $\% 20$ penal.pdf?sequence $=1 \&$ is Allowed $=\mathrm{y}$

Blázquez Entonado, F. (2001). Sociedad de la información y educación. Consejería de Educación, Ciencia y Tecnología, Dirección General de Ordenación, Renovación y Centros. Mérida.

Comisión Económica para América Latina y El Caribe. (2009). La sociedad de la información en América Latina y el Caribe. Editorial Naciones Unidas.

Díaz, F. (2010). Tecnologías de la información y la comunicación y nuevas dimensiones de los derechos fundamentales. Fondo Editorial PUCP.

González, J. (2015). Reflexiones sobre el proyecto de ley orgánica de modificación de la LECRIM para el fortalecimiento de las garantías procesales y la regulación de las medidas de investigación tecnológicas. Revista Electrónica de Ciencia Penal y Criminología, (17), 2-41. http://criminet.ugr.es/recpc/17/recpc17-06. pdf

Juan Gómez, M. (2015). 10 cosas que deberías saber de la reforma de la Ley de Enjuiciamiento Civil. Diario la Ley. https://diariolaley.laleynext.es/content/ Documento.aspx? params=H4sIAAAAAAAEAMtMSbF1CTEAAiMjC0MTC7Wy1KLizPw8WyMD Q1NDIAAJZKZVuuQnh1QWpNqmJeYUp6q1JuXnZ6Poi4erT0wqzs8pLUkNLcq0DSkqTVUrSExPDckPzkgsSrUFGlOam5pXkq-XWFxQAQAiNh3UfQAAAA==WKE

Montesinos, A. (2009). La videoconferencia como instrumento probatorio en el proceso penal. Marcial Pons.

Mozo, J. \& Nogueras, J. (2014). La digitalización de documentos en la Administración de Justicia. Ibersid, (8), 1-6. https://www.ibersid.eu/ojs/index.php/ibersid/article/ view/4179/3794

Pérez Luño, A. (2010). Nuevas tecnologías y nuevos derechos. En Efectos de las Tecnologías de la Información y la Comunicación sobre los Derechos Humanos (pp. 18-37). Instituto de Derechos Humanos de Catalunya.

Rivielo, A. (2006). El sistema telemático Lente. BOLETIC, 1-4. https:/www.astic.es/ sites/default/files/articulosboletic/mono3_1.pdf

Sotomarino Cáceres, S. (2010). Análisis del funcionamiento de las redes sociales facebook y twitter en relación con los derechos personales a la imagen y a la intimidad 
como a la institución de la responsabilidad civil [Trabajo de investigación, Universidad de San Martín de Porres]. https://www.yumpu.com/es/document/ $\mathrm{read} / 14252832 /$ analisis-del-funcionamiento-de-las-redes-sociales-facebook-ytwitter- 